Article

\title{
AN EXACT SOLUTION TO THE FREE VIBRATION ANALYSIS OF A UNIFORM TIMOSHENKO BEAM USING AN ANALYTICAL APPROACH
}

Author:

Valentin Fogang

\begin{abstract}
This study presents an analytical solution to the free vibration analysis of a uniform Timoshenko beam. The Timoshenko beam theory covers cases associated with small deflections based on shear deformation and rotary inertia considerations. A material law combining bending, shear, curvature, and natural frequency is presented. This complete study is based on this material law and closed form solutions are found. The free vibration response of single-span systems, as well as that of spring-mass systems, is analyzed. Closed-form formulations of matrices expressing the boundary conditions are presented; the natural frequencies are determined by solving the eigenvalue problem. First-order dynamic stiffness matrices in local coordinates are determined. Finally, second-order analysis of beams resting on an elastic Winkler foundation is conducted.
\end{abstract}

\section{Keywords}

Timoshenko beam; rotary inertia; bending-shear-curvature-natural frequency relationship; spring-mass system vibration; closed-form solutions; first-order dynamic stiffness matrix; second-order vibration analysis

Corresponding author:

$\begin{array}{ll} & \text { Valentin Fogang } \\ & \text { Civil Engineer } \\ & \text { C/o BUNS Sarl P.O Box 1130 Yaounde Cameroon } \\ \text { E-mail: } & \text { valentin.fogang@bunscameroun.com } \\ \text { ORCID iD } & \underline{\text { https://orcid.org/0000-0003-1256-9862 }}\end{array}$

\section{Introduction}

Free vibration analysis leads to the determination of the natural frequencies of structures. Natural frequencies are important for the analysis of the response of structures subjected to dynamic loadings such as wind, earthquake, traffic, etc. Various studies have focused on the free vibration analysis of Timoshenko beams. Kocatürk et al. [1] used Lagrange equations to examine the free vibration characteristics of Timoshenko beams. Gürgöze [2] used the Lagrange multiplier method to determine the free vibration characteristics of a cantilevered Euler-Bernoulli beam with a tip mass where a spring-mass system is attached to it. Yesilce et al. [3] studied the free vibration of a multi-span Timoshenko beam carrying multiple spring-mass systems; the natural frequencies were calculated by using the secant method and the mode shapes were presented in graphs. Ghannadiasl et al. [4] used the Green functions to analytically solve the case of beams with various boundary conditions resting on an elastic Winkler foundation and subjected to an axial load; the Green function method was utilized to evaluate the free vibration of the Timoshenko beam. Osadebe et al. [5] proposed a model for the free vibration analysis of a Timoshenko beam in which the finite element method was applied in conjunction with the energy method; the Timoshenko beam was divided into two virtual beams, namely, an Euler-Bernoulli beam and a shear layer beam. Kruszewski [6] presented a theoretical analysis of the effect of transverse shear and rotary inertia on the natural frequencies of a uniform cantilevered Timoshenko beam. In the present paper, an analytical solution to the free vibration analysis of a uniform Timoshenko beam is presented, together with a material law describing the relationship between the curvature, the bending moment, the bending stiffness, the shear force, the shear stiffness, and the natural frequency. Based on this material law, closed-form expressions of efforts and deformations are derived. The 
natural frequencies of single-span beams and beams with spring-mass systems are then determined by solving the eigenvalue problem.

\section{Materials and Methods}

\subsection{First-order vibration analysis}

The sign conventions adopted for bending moments, shear forces, and displacements are illustrated in Figure 1.

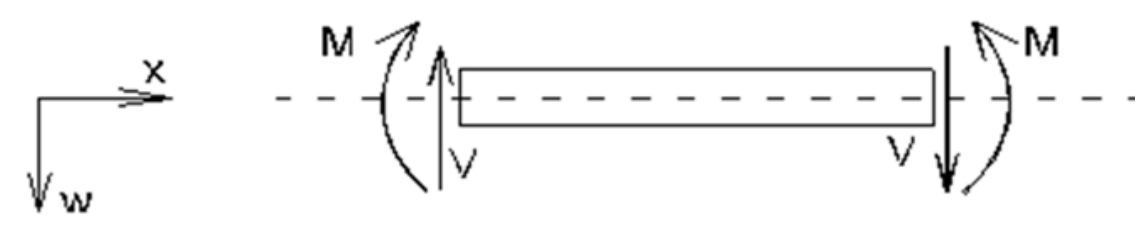

Figure 1. Sign conventions for bending moments, shear forces, and displacements.

For free vibration analysis, the balance of forces and moments applied on a Timoshenko beam element yields

$$
\begin{aligned}
& \frac{\partial V^{*}(x, t)}{\partial x}=\mu \frac{\partial^{2} w^{*}(x, t)}{\partial t^{2}} \\
& \frac{\partial M^{*}(x, t)}{\partial x}-V^{*}(x, t)=-\rho I \frac{\partial^{2} \varphi^{*}(x, t)}{\partial t^{2}}
\end{aligned}
$$

where $\mu$ is the beam's mass per unit length, $\rho$ is its mass per unit volume $(\mu=\rho A)$, A is the cross-sectional area, I is the second moment of area, $\mathrm{M}^{*}$ is the bending moment, $\mathrm{V}^{*}$ is the shear force, $\mathrm{w}^{*}$ is the beam deflection (in the positive downward direction), and $\varphi^{*}$ is the rotation of the cross section (positive in the clockwise direction).

According to the Timoshenko beam theory, the bending moment and the shear force are related to the deflection and the rotation of the cross section as follows.

$$
\begin{aligned}
& M^{*}(x, t)=-E I \frac{\partial \varphi^{*}(x, t)}{\partial x} \\
& V^{*}(x, t)=\kappa G A \times\left(\frac{\partial w^{*}(x, t)}{\partial x}-\varphi^{*}(x, t)\right)
\end{aligned}
$$

Differentiating Equation (2) with respect to $x$, and combining the result with Equations (1) and (3) yields

$$
\frac{\partial^{2} M^{*}(x, t)}{\partial x^{2}}-\mu \frac{\partial^{2} w^{*}(x, t)}{\partial t^{2}}-\frac{\rho}{E} \frac{\partial^{2} M^{*}(x, t)}{\partial t^{2}}=0
$$

A harmonic vibration being assumed, $\mathrm{M}^{*}(\mathrm{x}, \mathrm{t}), \mathrm{V}^{*}(\mathrm{x}, \mathrm{t}), \mathrm{w}^{*}(\mathrm{x}, \mathrm{t})$, and $\varphi^{*}(\mathrm{x}, \mathrm{t})$ can be expressed as follows:

$$
\begin{aligned}
& w^{*}(x, t)=w(x) \times \sin (\omega t+\theta) \\
& \varphi^{*}(x, t)=\varphi(x) \times \sin (\omega t+\theta)
\end{aligned}
$$




$$
\begin{aligned}
& M^{*}(x, t)=M(x) \times \sin (\omega t+\theta) \\
& V^{*}(x, t)=V(x) \times \sin (\omega t+\theta)
\end{aligned}
$$

Here, $\omega$ is the circular frequency of the beam. Substituting Equations (6a) and (6c) into Equation (5) yields

$$
\frac{d^{2} M(x)}{d x^{2}}+\frac{\rho \omega^{2}}{E} M(x)+\mu \omega^{2} w(x)=0
$$

The application of Equations (6a), (6c), and (6d) to Equations (3) and (4) with rearrangement yields

$$
\begin{aligned}
& \frac{d w(x)}{d x}=\varphi(x)+\frac{V(x)}{\kappa G A}, \\
& M(x)=-E I \frac{d \varphi(x)}{d x} .
\end{aligned}
$$

Substituting Equations (6b), (6c), and (6d) into Equation (2) yields

$$
\frac{d M(x)}{d x}-V(x)=\rho I \omega^{2} \times \varphi(x)
$$

The combination of Equations (8) and (10) yields

$$
\begin{aligned}
& \left(1-\frac{\rho I \omega^{2}}{\kappa G A}\right) \times V(x)=\frac{d M(x)}{d x}-\rho I \omega^{2} \frac{d w(x)}{d x}, \\
& \left(\rho I \omega^{2}-\kappa G A\right) \times \varphi(x)=\frac{d M(x)}{d x}-\kappa G A \frac{d w(x)}{d x} .
\end{aligned}
$$

Differentiating both sides of Equation (8) with respect to $x$ yields

$$
\frac{d^{2} w(x)}{d x^{2}}=\frac{d \varphi(x)}{d x}+\frac{1}{\kappa G A} \times \frac{d V(x)}{d x} .
$$

Substituting Equations (9) and (11) into Equation (13) and rearranging yields the following material law which combines bending, shear, curvature, and natural frequency:

$$
\frac{d^{2} w(x)}{d x^{2}}+\left(1-\frac{\rho I \omega^{2}}{\kappa G A}\right) \times \frac{M(x)}{E I}-\frac{1}{\kappa G A} \times \frac{d^{2} M(x)}{d x^{2}}=0
$$

The coefficient of free vibration is denoted by $\phi$, the coefficient of rotary inertia by $k_{\mathrm{RI}}$, and the bending shear factor by $\alpha$.

$$
\begin{aligned}
& \omega^{2}=\phi E I /\left(\mu l^{4}\right) \\
& k_{R I}=I /\left(A l^{2}\right) \\
& \alpha=E I /\left(\kappa G A l^{2}\right)
\end{aligned}
$$


Applying Equations (15a) to (15c) to Equations (7) and (14) yields

$$
\begin{aligned}
& \frac{d^{2} M(x)}{d x^{2}}+\phi k_{R I} \frac{1}{l^{2}} M(x)+\phi E I \frac{1}{l^{4}} w(x)=0 \\
& E I \frac{d^{2} w(x)}{d x^{2}}+\left(1-\phi \alpha k_{R I}\right) M(x)-\alpha l^{2} \frac{d^{2} M(x)}{d x^{2}}=0 .
\end{aligned}
$$

Differentiating Equation (16) twice with respect to $x$ and combining it with Equation (17) yields

$$
\frac{d^{4} M(x)}{d x^{4}}+\phi\left(\alpha+k_{R I}\right) \frac{1}{l^{2}} \times \frac{d^{2} M(x)}{d x^{2}}-\phi\left(1-\phi \alpha k_{R I}\right) \frac{1}{l^{4}} M(x)=0 \text {. }
$$

The application of Equations (15a) to (15c) to Equations (7), (11) and (12) yields

$$
\begin{aligned}
& \phi E I / l^{2} \times w(x)=-l^{2} \frac{d^{2} M(x)}{d x^{2}}-\phi k_{R I} M(x) \\
& \phi E I / l^{2} \times\left(\phi \alpha k_{R I}-1\right) \times l \varphi(x)=\phi\left(\alpha+k_{R I}\right) l \frac{d M(x)}{d x}+l^{3} \frac{d^{3} M(x)}{d x^{3}}, \\
& \left(1-\phi \alpha k_{R I}\right) \times l V(x)=\left(1+\phi k_{R I}^{2}\right) l \frac{d M(x)}{d x}+k_{R I} l^{3} \frac{d^{3} M(x)}{d x^{3}}
\end{aligned}
$$

The solution of Equation (18) for low-frequency modes $\left(1-\phi \alpha \mathrm{k}_{\mathrm{RI}} \geq 0\right)$ is as follows:

$$
\begin{aligned}
& M(x)=A_{1} \cosh \xi_{1} \frac{x}{l}+B_{1} \sinh \xi_{1} \frac{x}{l}+C_{1} \cos \xi_{2} \frac{x}{l}+D_{1} \sin \xi_{2} \frac{x}{l} \\
& \xi_{1}=\sqrt{\frac{-\phi\left(\alpha+k_{R I}\right)+\sqrt{\phi^{2}\left(\alpha+k_{R I}\right)^{2}+4 \phi\left(1-\phi \alpha k_{R I}\right)}}{2}} \\
& \xi_{2}=\sqrt{\frac{\phi\left(\alpha+k_{R I}\right)+\sqrt{\phi^{2}\left(\alpha+k_{R I}\right)^{2}+4 \phi\left(1-\phi \alpha k_{R I}\right)}}{2}}
\end{aligned}
$$

with integration constants $\mathrm{A}_{1}, \mathrm{~B}_{1}, \mathrm{C}_{1}$, and $\mathrm{D}_{1}$.

It is noted that $\phi^{2}\left(\alpha+k_{R I}\right)^{2}+4 \phi\left(1-\phi \alpha k_{R I}\right)=\phi\left[\phi\left(\alpha-k_{R I}\right)^{2}+4\right] \geq 0$

The application of Equations (22), (23), and (24) to Equations (19), (20), and (21) yields the following expressions for the deflection, the rotation of the cross section, and the shear force.

$$
\begin{aligned}
& \phi E I / l^{2} \times w(x)=-A_{1}\left(\xi_{1}^{2}+\phi k_{R I}\right) \cosh \xi_{1} \frac{x}{l}-B_{1}\left(\xi_{1}^{2}+\phi k_{R I}\right) \sinh \xi_{1} \frac{x}{l}+ \\
& C_{1}\left(\xi_{2}^{2}-\phi k_{R I}\right) \cos \xi_{2} \frac{x}{l}+D_{1}\left(\xi_{2}{ }^{2}-\phi k_{R I}\right) \sin \xi_{2} \frac{x}{l}
\end{aligned}
$$




$$
\begin{aligned}
& \phi E I / I^{2} \times\left(\phi \alpha k_{R I}-1\right) \times l \varphi(x)=\xi_{1}\left(\xi_{1}^{2}+\phi k_{R I}+\phi \alpha\right) A_{1} \sinh \xi_{1} \frac{x}{l}+ \\
& \xi_{1}\left(\xi_{1}^{2}+\phi k_{R I}+\phi \alpha\right) B_{1} \cosh \xi_{1} \frac{x}{l}+\xi_{2}\left(\xi_{2}^{2}-\phi k_{R I}-\phi \alpha\right) C_{1} \sin \xi_{2} \frac{x}{l} \\
& +\xi_{2}\left(-\xi_{2}^{2}+\phi k_{R I}+\phi \alpha\right) D_{1} \cos \xi_{2} \frac{x}{l} \\
& \left(1-\phi \alpha k_{R I}\right) \times l V(x)=\xi_{1}\left(1+\phi k_{R I}^{2}+k_{R I} \xi_{1}^{2}\right) A_{1} \sinh \xi_{1} \frac{x}{l}+ \\
& \xi_{1}\left(1+\phi k_{R I}^{2}+k_{R I} \xi_{1}^{2}\right) B_{1} \cosh \xi_{1} \frac{x}{l}+\xi_{2}\left(-1-\phi k_{R I}^{2}+k_{R I} \xi_{2}^{2}\right) C_{1} \sin \xi_{2} \frac{x}{l} \\
& +\xi_{2}\left(1+\phi k_{R I}^{2}-k_{R I} \xi_{2}^{2}\right) D_{1} \cos \xi_{2} \frac{x}{l}
\end{aligned}
$$

The application of Equations (27a), (27b), and (27c) to Equations (19), (20), and (21) yields the deflection, the rotation of the cross section, and the shear force.

\subsection{First-order vibration analysis of a beam with a mass, spring, or spring-mass system at its end}

The stiffness of the spring is $\mathrm{Kp}$, and the concentrated mass is $\mathrm{Mp}$. We set

$$
\begin{aligned}
& K_{p}=k_{p}^{*} \times E I / l^{3}, \\
& M_{p}=m_{p}^{*} \times \mu l, \\
& W=E I / l^{2} \times W .
\end{aligned}
$$

We analyze here the case of a cantilevered beam carrying a tip mass at the beam's end, as represented in Figure 2 . 


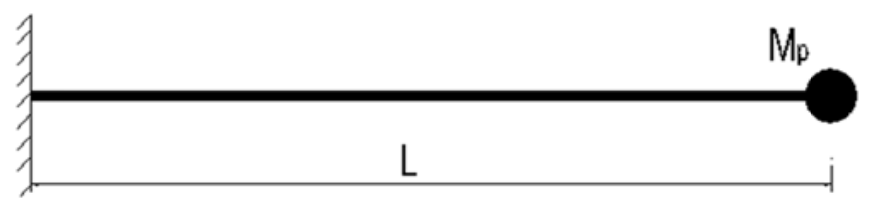

Figure 2. Cantilevered beam with a tip mass.

At the beam's end with the mass, the bending moment is $\mathrm{M}_{\mathrm{i}}=0$. The balance of vertical forces (combined with Equations (15a), (28b), and (28c)) yields

$$
V_{i}-M_{p} \omega^{2} \times w_{i}=0 \rightarrow l V_{i}-m_{p}^{*} \phi W_{i}=0
$$

Let us now analyze a cantilevered beam with a tip mass and spring support as shown in Figure 3.

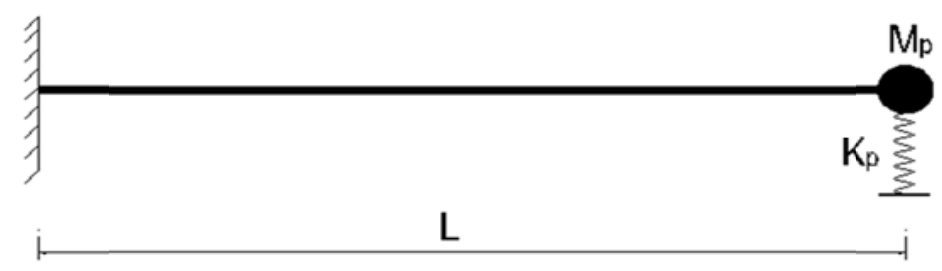

Figure 3. Cantilevered beam with a tip mass and spring support.

At the beam's end with the mass, the bending moment is $\mathrm{M}_{\mathrm{i}}=0$. The balance of vertical forces (combined with Equations (15a), (28a), (28b), and (28c)) yields,

$$
V_{i}+K_{p} w_{i}-M_{p} \omega^{2} \times w_{i}=0 \rightarrow l V_{i}+\left(k_{p}^{*}-m_{p}^{*} \phi\right) W_{i}=0 .
$$

Let us now analyze a cantilevered beam carrying a spring-mass system at the beam's end, as represented in Figure 4 . The deflection of the mass is denoted by $\mathrm{w}_{\mathrm{iM}}$.

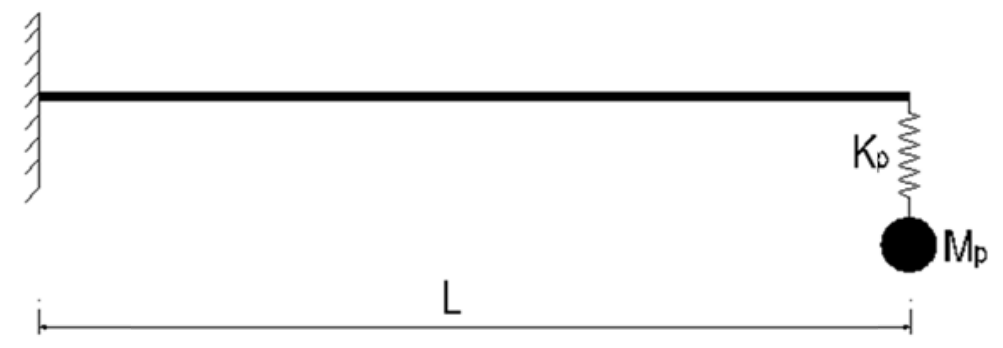

Figure 4. Cantilevered beam with a spring-mass system.

At the beam's end with the mass, the bending moment is $\mathrm{M}_{\mathrm{i}}=0$. The balance of vertical forces (combined with Equations (15a), (28a), (28b), and (28c)) yields

$$
\begin{aligned}
& V_{i}-M_{p} \omega^{2} \times w_{i M}=0 \rightarrow l V_{i}-m_{p}{ }^{*} \phi W_{i M}=0 \\
& M_{p} \omega^{2} \times w_{i M}=K_{p} \times\left(w_{i M}-w_{i}\right) \rightarrow\left(k_{p}{ }^{*}-m_{p}{ }^{*} \phi\right) W_{i M}=k_{p}{ }^{*} W_{i}
\end{aligned}
$$




\subsection{First-order vibration analysis of a beam with an intermediate mass, spring, or spring-mass system}

The transition equations express the continuity of the deflection and rotation of the cross section (Equations (25) and (26)) and the equilibrium of the bending moment and the balance of vertical forces (Equations (22) and (27)).

In case of a mass on a spring support, the transition equations are as follows.

$$
\begin{aligned}
& w_{i l}=w_{i r} \rightarrow \phi E I / l^{2} \times w_{i l}=\phi E I / l^{2} \times w_{i r} \\
& \varphi_{i l}=\varphi_{i r} \rightarrow \phi E I / l^{2} \times\left(\phi \alpha k_{R I}-1\right) \times l \varphi_{i l}=\phi E I / l^{2} \times\left(\phi \alpha k_{R I}-1\right) \times l \varphi_{i r} \\
& M_{i l}=M_{i r} \\
& V_{i l}-V_{i r}+K_{p} w_{i r}-M_{p} \omega^{2} \times w_{i}=0 \rightarrow l V_{i l}-l V_{i r}+\left(k_{p}^{*}-m_{p}^{*} \phi\right) W_{i r}=0
\end{aligned}
$$

In case of a concentrated mass, Equations (32a), (32b), and (32c) apply. The balance of vertical forces yields

$$
V_{i l}-V_{i r}-M_{p} \omega^{2} \times w_{i r}=0 \rightarrow l V_{i l}-l V_{i r}-m_{p}^{*} \phi W_{i r}=0
$$

In case of a spring-mass system, Equations (32a), (32b), and (32c) apply. The balance of vertical forces yields

$$
\begin{aligned}
& V_{i l}-V_{i r}-M_{p} \omega^{2} \times w_{i M}=0 \rightarrow l V_{i l}-l V_{i r}-m_{p}^{*} \phi W_{i M}=0 \\
& M_{p} \omega^{2} \times w_{i M}=K_{p} \times\left(w_{i M}-w_{i r}\right) \rightarrow\left(k_{p}{ }^{*}-m_{p}{ }^{*} \phi\right) W_{i M}=k_{p}^{*} W_{i r}
\end{aligned}
$$

\subsection{First-order dynamic stiffness matrix of the Timoshenko beam}

The analysis conducted here applies for $1-\phi \alpha \mathrm{k}_{\mathrm{RI}} \geq 0$ (low-frequency modes).

The sign conventions for bending moments, shear forces, displacements, and rotations adopted for use in determining the element stiffness matrix in local coordinates are illustrated in Figure 5.

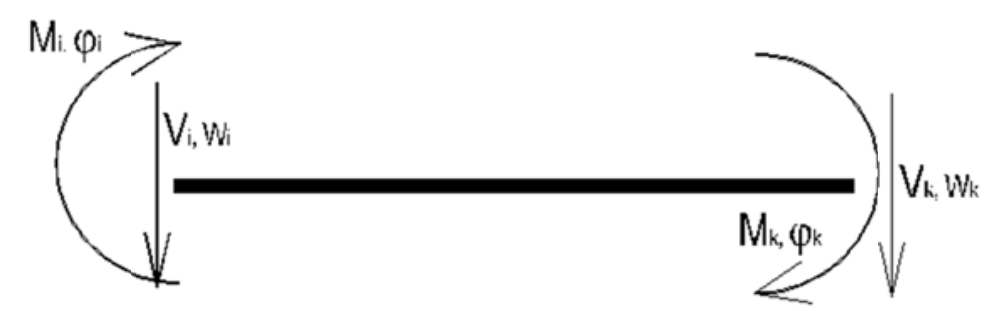

Figure 5. Sign conventions for moments, shear forces, displacements, and rotations for the stiffness matrix

Let us define the following vectors,

$$
\begin{aligned}
& \vec{S}=\left[V_{i} ; M_{i} ; V_{k} ; M_{k}\right]^{T} \\
& \vec{V}=\left[w_{i} ; \varphi_{i} ; w_{k} ; \varphi_{k}\right]^{T}
\end{aligned}
$$


The dynamic element stiffness matrix in local coordinates of the Timoshenko beam is denoted by $\mathrm{K}_{\mathrm{Tb}, \mathrm{dyn}}$. The relationship between the aforementioned vectors is as follows:

$$
\vec{S}=K_{T b, d y n} \times \vec{V}
$$

Considering the sign conventions adopted for bending moments and shear forces in general (see Figure 1) and for bending moments and shear forces in the element stiffness matrix (see Figure 5), we can set the following static compatibility boundary conditions:

$$
\begin{aligned}
& V_{i}=-V(x=0) \\
& M_{i}=M(x=0) \\
& V_{k}=V(x=l) \\
& M_{k}=-M(x=l)
\end{aligned}
$$

Considering the sign conventions adopted for the displacements and rotations in general (see Figure 1) and for displacements and rotations in the member stiffness matrix (see Figure 5), we can set the following geometric compatibility boundary conditions:

$$
\begin{aligned}
& \omega(x=0)=w_{i} \\
& \varphi(x=0)=\varphi_{i} \\
& \omega(x=l)=w_{k} \\
& \varphi(x=l)=\varphi_{k}
\end{aligned}
$$

The application of Equations (22) and (27) to Equations (36a) to (36d) yields Equation (38) below.

$$
\begin{aligned}
& {\left[\begin{array}{cccc}
-\left(1-\phi \alpha k_{R I}\right) l & 0 & 0 & 0 \\
0 & 1 & 0 & 0 \\
0 & 0 & \left(1-\phi \alpha k_{R I}\right) l & 0 \\
0 & 0 & 0 & -1
\end{array}\right] \times\left[\begin{array}{c}
V_{i} \\
M_{i} \\
V_{k} \\
M_{k}
\end{array}\right]=} \\
& {\left[\begin{array}{cccc}
0 & \xi_{1}\left(1+\phi k_{R I}^{2}+k_{R I} \xi_{1}^{2}\right) & 0 & \xi_{2}\left(1+\phi k_{R I}^{2}-k_{R I} \xi_{2}^{2}\right) \\
1 & 0 & 1 & 0 \\
\xi_{1}\left(1+\phi k_{R I}^{2}+k_{R I} \xi_{1}^{2}\right) & \xi_{1}\left(1+\phi k_{R I}^{2}+k_{R I} \xi_{1}^{2}\right) & \xi_{2}\left(-1-\phi k_{R I}^{2}+k_{R I} \xi_{2}^{2}\right) & +\xi_{2}\left(1+\phi k_{R I}{ }^{2}-k_{R I} \xi_{2}^{2}\right) \\
\times \sinh \xi_{1} & \times \cosh \xi_{1} & \times \sin \xi_{2} & \times \cos \xi_{2} \\
\cosh \xi_{1} & \sinh \xi_{1} & \cos \xi_{2} & \sin \xi_{2}
\end{array}\right] \times\left[\begin{array}{c}
A_{1} \\
B_{1} \\
C_{1} \\
D_{1}
\end{array}\right]}
\end{aligned}
$$


The application of Equations (25) and (26) to Equations (37a) to (37d) yields Equation (39) below.

$\phi \frac{E I}{l^{2}} \times\left[\begin{array}{cccc}1 & 0 & 0 & 0 \\ 0 & l\left(\phi \alpha k_{R I}-1\right) & 0 & 0 \\ 0 & 0 & 1 & 0 \\ 0 & 0 & 0 & l\left(\phi \alpha k_{R I}-1\right)\end{array}\right] \times\left[\begin{array}{c}w_{i} \\ \varphi_{i} \\ w_{k} \\ \varphi_{k}\end{array}\right]=$

$$
\left[\begin{array}{cccc}
-\left(\xi_{1}^{2}+\phi k_{R I}\right) & 0 & \left(\xi_{2}^{2}-\phi k_{R I}\right) & \multicolumn{1}{c}{0} \\
0 & \xi_{1}\left(\xi_{1}^{2}+\phi k_{R I}+\phi \alpha\right) & 0 & \xi_{2}\left(-\xi_{2}^{2}+\phi k_{R I}+\phi \alpha\right) \\
-\left(\xi_{1}^{2}+\phi k_{R I}\right) \cosh \xi_{1} & -\left(\xi_{1}^{2}+\phi k_{R I}\right) \sinh \xi_{1} & \left(\xi_{2}^{2}-\phi k_{R I}\right) \cos \xi_{2} & \left(\xi_{2}^{2}-\phi k_{R I}\right) \sin \xi_{2} \\
\xi_{1}\left(\xi_{1}^{2}+\phi k_{R I}+\phi \alpha\right) & \xi_{1}\left(\xi_{1}^{2}+\phi k_{R I}+\phi \alpha\right) & \xi_{2}\left(\xi_{2}^{2}-\phi k_{R I}-\phi \alpha\right) & \xi_{2}\left(-\xi_{2}^{2}+\phi k_{R I}+\phi \alpha\right) \\
\times \sinh \xi_{1} & \times \cosh \xi_{1} & \times \sin \xi_{2} & \times \cos \xi_{2}
\end{array}\right] \times\left[\begin{array}{l}
A_{1} \\
B_{1} \\
C_{1} \\
D_{1}
\end{array}\right]
$$

Converting Equations (38) and (39) to matrix notation yields

$$
\begin{aligned}
& T_{S} \times \vec{S}=M_{S} \times \vec{C} \\
& \phi \frac{E I}{l^{2}} \times T_{V} \times \vec{V}=M_{V} \times \vec{C}
\end{aligned}
$$

where the vector $\vec{C}$ and the matrices $\boldsymbol{T}_{\mathrm{S}}, \boldsymbol{M}_{\mathbf{S}}, \boldsymbol{M}_{\mathrm{V}}$, and $\boldsymbol{T}_{\mathbf{V}}$ are defined as follows in Equations (41a) to (41e).

$$
\begin{aligned}
& \vec{C}=\left[A_{1} ; B_{1} ; C_{1} ; D_{1}\right]^{T} \\
& T_{S}=\left[\begin{array}{cccc}
-\left(1-\phi \alpha k_{R I}\right) l & 0 & 0 & 0 \\
0 & 1 & 0 & 0 \\
0 & 0 & \left(1-\phi \alpha k_{R I}\right) l & 0 \\
0 & 0 & 0 & -1
\end{array}\right]
\end{aligned}
$$

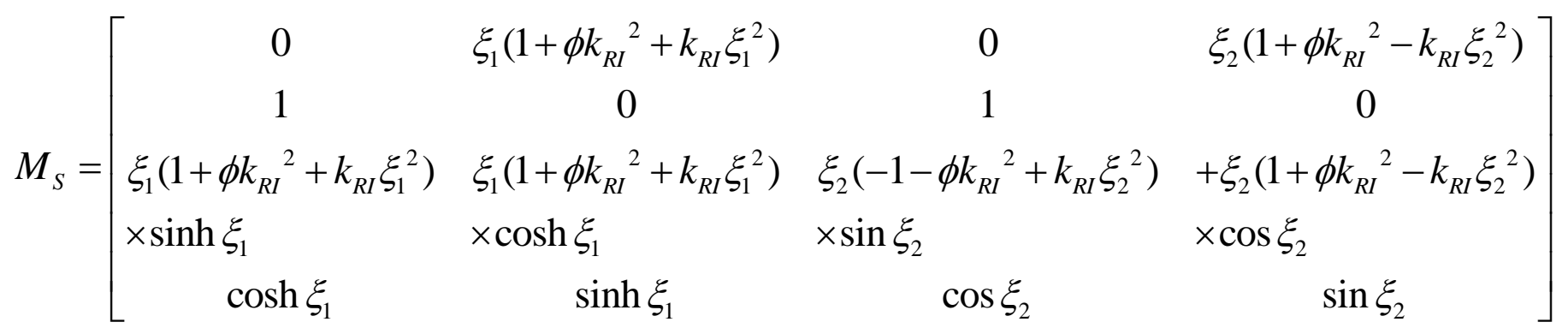




$$
\begin{aligned}
& T_{V}=\left[\begin{array}{cccc}
1 & 0 & 0 & 0 \\
0 & l\left(\phi \alpha k_{R I}-1\right) & 0 & 0 \\
0 & 0 & 1 & 0 \\
0 & 0 & 0 & l\left(\phi \alpha k_{R I}-1\right)
\end{array}\right] \\
& M_{V}=\left[\begin{array}{cccc}
-\left(\xi_{1}^{2}+\phi k_{R I}\right) & 0 & \left(\xi_{2}^{2}-\phi k_{R I}\right) & 0 \\
0 & \xi_{1}\left(\xi_{1}^{2}+\phi k_{R I}+\phi \alpha\right) & 0 & \xi_{2}\left(-\xi_{2}^{2}+\phi k_{R I}+\phi \alpha\right) \\
-\left(\xi_{1}^{2}+\phi k_{R I}\right) \cosh \xi_{1} & -\left(\xi_{1}^{2}+\phi k_{R I}\right) \sinh \xi_{1} & \left(\xi_{2}^{2}-\phi k_{R I}\right) \cos \xi_{2} & \left(\xi_{2}^{2}-\phi k_{R I}\right) \sin \xi_{2} \\
\xi_{1}\left(\xi_{1}^{2}+\phi k_{R I}+\phi \alpha\right) & \xi_{1}\left(\xi_{1}^{2}+\phi k_{R I}+\phi \alpha\right) & \xi_{2}\left(\xi_{2}^{2}-\phi k_{R I}-\phi \alpha\right) & \xi_{2}\left(-\xi_{2}^{2}+\phi k_{R I}+\phi \alpha\right) \\
\times \sinh \xi_{1} & \times \cosh \xi_{1} & \times \sin \xi_{2} & \times \cos \xi_{2}
\end{array}\right]
\end{aligned}
$$

The combination of Equations (35c), (40a), and (40b) yields

$$
K_{T b, d y n}=\phi \frac{E I}{l^{2}} \times T_{S}^{-1} \times M_{S} \times M_{V}^{-1} \times T_{V}
$$

\subsection{First-order vibration analysis of a beam resting on a Winkler foundation}

The stiffness of the Winkler foundation is $\mathrm{K}_{\mathrm{w}}$. We set

$$
K_{w}=k_{w}^{*} \times E I / l^{4} .
$$

Equation (1) then becomes

$$
\frac{\partial V^{*}(x, t)}{\partial x}-K_{w} w^{*}(x, t)=\mu \frac{\partial^{2} w^{*}(x, t)}{\partial t^{2}}
$$

The analysis continues similarly to that in Section 2.1. Thus, Equation (18) becomes

$$
\frac{d^{4} M(x)}{d x^{4}}+\left[\phi\left(\alpha+k_{R I}\right)-k_{w}{ }^{*} \alpha\right] \frac{1}{l^{2}} \frac{d^{2} M(x)}{d x^{2}}+\left(1-\phi \alpha k_{R I}\right)\left(k_{w}{ }^{*}-\phi\right) \frac{1}{l^{4}} M(x)=0
$$

The solution of Equation (45) yields the formulation of $\mathrm{M}(\mathrm{x})$ with four integration constants. The deflection $\mathrm{w}(\mathrm{x})$, the rotation of the cross section $\varphi(x)$, and the shear force are determined as follows.

$$
\begin{aligned}
& E I / l^{2} \times\left(\phi-k_{w}^{*}\right) \times w(x)=-l^{2} \frac{d^{2} M(x)}{d x^{2}}-\phi \\
& E I / l^{2} \times\left(\phi \alpha k_{R I}-1\right) \times\left(\phi-k_{w}^{*}\right) \times l \varphi(x)= \\
& {\left[\phi\left(\alpha+k_{R I}\right)-k_{w}^{*} \alpha\right] l \frac{d M(x)}{d x}+l^{3} \frac{d^{3} M(x)}{d x^{3}}}
\end{aligned}
$$


$\left(1-\phi \alpha k_{R I}\right)\left(\phi-k_{w}^{*}\right) \times l V(x)=\left(\phi+\phi^{2} k_{R I}^{2}-k_{w}^{*}\right) l \frac{d M(x)}{d x}+\phi k_{R I} l^{3} \frac{d^{3} M(x)}{d x^{3}}$

\subsection{Second-order vibration analysis of a beam resting on a Winkler foundation}

The axial force $\mathrm{N}$ is assumed to be constant.

When the shear force is replaced by the transverse force $T^{*}(x)$, Equations (1) and (2) become

$$
\begin{aligned}
& \frac{\partial T^{*}(x, t)}{\partial x}-K_{w} w^{*}(x, t)=\mu \frac{\partial^{2} w^{*}(x, t)}{\partial t^{2}} \\
& \frac{\partial M^{*}(x, t)}{\partial x}+N \frac{\partial w^{*}(x, t)}{\partial x}-T^{*}(x, t)=-\rho I \frac{\partial^{2} \varphi^{*}(x, t)}{\partial t^{2}}
\end{aligned}
$$

The transverse force - shear force relationship is as follows:

$$
T(x)=V(x)+N \frac{d w(x)}{d x} .
$$

Differentiating Equation (50) with respect to $x$ and combining it with Equations (49) and (3) yields

$$
\frac{\partial^{2} M^{*}(x, t)}{\partial x^{2}}+N \frac{\partial^{2} w^{*}(x, t)}{\partial x^{2}}-\mu \frac{\partial^{2} w^{*}(x, t)}{\partial t^{2}}-\frac{\rho}{E} \frac{\partial^{2} M^{*}(x, t)}{\partial t^{2}}-K_{w} w^{*}(x, t)=0
$$

The separation of variables (Equations (6a) to (6d)) applied to Equations (52) and (50) yields

$$
\begin{aligned}
& \frac{d^{2} M(x)}{d x^{2}}+N \frac{d^{2} w(x)}{d x^{2}}+\frac{\rho \omega^{2}}{E} M(x)+\left(\mu \omega^{2}-K_{w}\right) \times w(x)=0, \\
& \frac{d M(x)}{d x}+N \frac{d w(x)}{d x}-T(x)=\rho I \omega^{2} \times \varphi(x) .
\end{aligned}
$$

Substituting Equation (51) into Equation (54) yields

$$
\frac{d M(x)}{d x}-V(x)=\rho I \omega^{2} \times \varphi(x)
$$

Equations (55) and (10) are identical. The combination of Equations (8) and (55) yields Equations (11), (12), (13), and (14). The combination of Equations (11) and (51) yields,

$$
\left(1-\frac{\rho I \omega^{2}}{\kappa G A}\right) \times T(x)=\frac{d M(x)}{d x}+\left[N\left(1-\frac{\rho I \omega^{2}}{\kappa G A}\right)-\rho I \omega^{2}\right] \times \frac{d w(x)}{d x} .
$$

The combination of Equations (8) and (55) yields

$$
\left(\rho I \omega^{2}-\kappa G A\right) \times \varphi(x)=\frac{d M(x)}{d x}-\kappa G A \times \frac{d w(x)}{d x} .
$$

The combination of Equations (14) and (53) yields 


$$
\left(\mu \omega^{2}-K_{w}\right) \times w(x)=-\left(1+\frac{N}{\kappa G A}\right) \frac{d^{2} M(x)}{d x^{2}}-\left(\frac{\rho \omega^{2}}{E}-\left(1-\frac{\rho I \omega^{2}}{\kappa G A}\right) \frac{N}{E I}\right) M(x) .
$$

Differentiating both sides of Equation (58) twice with respect to $x$ and combining the result with Equation (14) yields

$$
\begin{aligned}
& \left(1+\frac{N}{\kappa G A}\right) \frac{d^{4} M(x)}{d x^{4}}+\left(\frac{\rho \omega^{2}}{E}-\left(1-\frac{\rho I \omega^{2}}{\kappa G A}\right) \frac{N}{E I}-\frac{K_{w}-\mu \omega^{2}}{\kappa G A}\right) \frac{d^{2} M(x)}{d x^{2}}+ \\
& \left(1-\frac{\rho I \omega^{2}}{\kappa G A}\right) \frac{K_{w}-\mu \omega^{2}}{E I} M(x)=0
\end{aligned}
$$

The axial force $\mathrm{N}$ can be expressed as follows:

$$
N=k \times E I / l^{2} .
$$

Substituting Equations (15a), (15b), (15c), (43), and (60) into Equation (59) yields

$$
\begin{aligned}
& (1+k \alpha) \frac{d^{4} M(x)}{d x^{4}}+\left[-k_{w}^{*} \alpha+\phi\left(\alpha+k_{R I}\right)-k\left(1-\phi \alpha k_{R I}\right)\right] \frac{1}{l^{2}} \frac{d^{2} M(x)}{d x^{2}}+ \\
& \left(1-\phi \alpha k_{R I}\right)\left(k_{w}{ }^{*}-\phi\right) \frac{1}{l^{4}} M(x)=0
\end{aligned}
$$

The solution of Equation (61) yields the formulation of $\mathrm{M}(\mathrm{x})$ with four integration constants. Equations (58), (57), and (56) are used to determine the deflection $\mathrm{w}(\mathrm{x})$, the rotation of the cross section $\varphi(\mathrm{x})$, and the transverse force as follows.

$$
\begin{aligned}
& E I / l^{2} \times\left(\phi-k_{w}^{*}\right) \times w(x)=-(1+k \alpha) l^{2} \frac{d^{2} M(x)}{d x^{2}}-\left[\phi k_{R I}-k\left(1-\phi \alpha k_{R I}\right)\right] M(x) \\
& E I / l^{2} \times\left(\phi \alpha k_{R I}-1\right) \times\left(\phi-k_{w}^{*}\right) \times l \varphi(x)= \\
& {\left[\phi\left(\alpha+k_{R I}\right)-k_{w}^{*} \alpha-k\left(1-\phi \alpha k_{R I}\right)\right] l \frac{d M(x)}{d x}+(1+k \alpha) l^{3} \frac{d^{3} M(x)}{d x^{3}}} \\
& \left(1-\phi \alpha k_{R I}\right) \times\left(k_{w}{ }^{*}-\phi\right) \times l T(x)=\left[k_{w}{ }^{*}-\phi-\left[k\left(1-\phi \alpha k_{R I}\right)-\phi k_{R I}\right]^{2}\right] l \frac{d M(x)}{d x}+ \\
& {\left[k\left(1-\phi \alpha k_{R I}\right)-\phi k_{R I}\right](1+k \alpha) l^{3} \frac{d^{3} M(x)}{d x^{3}}}
\end{aligned}
$$

Equations (61), (62), (63), and (64) depend on the following parameters: 


$$
\begin{aligned}
& A=1+k \alpha \\
& B=-k_{w}{ }^{*} \alpha+\phi\left(\alpha+k_{R I}\right)-k\left(1-\phi \alpha k_{R I}\right) \\
& C=\left(1-\phi \alpha k_{R I}\right)\left(k_{w}{ }^{*}-\phi\right) \\
& \Delta=B^{2}-4 A C \\
& E=\phi k_{R I}-\left(1-\phi \alpha k_{R I}\right) k
\end{aligned}
$$

The following cases are analyzed herein.

Case 1:

$$
\begin{aligned}
& \Delta>\mathbf{0} \quad \mathbf{- B / A}>\mathbf{0} \quad \mathbf{C} / \mathbf{A}>\mathbf{0} \\
& M(x)=A_{1} \cosh \xi_{1} \frac{x}{l}+B_{1} \sinh \xi_{1} \frac{x}{l}+C_{1} \cosh \xi_{2} \frac{x}{l}+D_{1} \sinh \xi_{2} \frac{x}{l} \\
& \xi_{1,2}=\sqrt{(-B \pm \sqrt{\Delta}) / 2 A}
\end{aligned}
$$

Case 2:

$$
\begin{aligned}
& \Delta>0 \quad \mathrm{C} / \mathrm{A}<0 \\
& M(x)=A_{1} \cosh \xi_{1} \frac{x}{l}+B_{1} \sinh \xi_{1} \frac{x}{l}+C_{1} \cos \xi_{2} \frac{x}{l}+D_{1} \sin \xi_{2} \frac{x}{l} \\
& \xi_{1,2}=\sqrt{(\mp B+\sqrt{\Delta}) / 2 A}
\end{aligned}
$$

Case 3:

$$
\begin{aligned}
& \Delta>\mathbf{0} \quad \mathbf{- B} / \mathbf{A}<\mathbf{0} \quad \mathbf{C} / \mathbf{A}>\mathbf{0} \\
& M(x)=A_{1} \cos \xi_{1} \frac{x}{l}+B_{1} \sin \xi_{1} \frac{x}{l}+C_{1} \cos \xi_{2} \frac{x}{l}+D_{1} \sin \xi_{2} \frac{x}{l} \\
& \xi_{1,2}=\sqrt{(B \pm \sqrt{\Delta}) / 2 A}
\end{aligned}
$$

Case 4: $\Delta=0 \quad-B / A>0$

$$
\begin{aligned}
& M(x)=A_{1} \cosh \xi_{1} \frac{x}{l}+B_{1} \xi_{1} \frac{x}{l} \cosh \xi_{1} \frac{x}{l}+C_{1} \sinh \xi_{1} \frac{x}{l}+D_{1} \xi_{1} \frac{x}{l} \sinh \xi_{1} \frac{x}{l} \\
& \xi_{1}=\sqrt{-B / 2 A}
\end{aligned}
$$

Case 5: $\quad \Delta=0 \quad-\mathrm{B} / \mathrm{A}<0$

$$
\begin{aligned}
& M(x)=A_{1} \cos \xi_{1} \frac{x}{l}+B_{1} \xi_{1} \frac{x}{l} \cos \xi_{1} \frac{x}{l}+C_{1} \sin \xi_{1} \frac{x}{l}+D_{1} \xi_{1} \frac{x}{l} \sin \xi_{1} \frac{x}{l} \\
& \xi_{1}=\sqrt{B / 2 A}
\end{aligned}
$$

Case 6: $\Delta<0$

$$
\begin{aligned}
& \Delta<0 \\
& M(x)=A_{1} \cosh \xi_{1} \frac{x}{l} \times \cos \xi_{2} \frac{x}{l}+B_{1} \cosh \xi_{1} \frac{x}{l} \times \sin \xi_{2} \frac{x}{l}+ \\
& C_{1} \sinh \xi_{1} \frac{x}{l} \times \cos \xi_{2} \frac{x}{l}+D_{1} \sinh \xi_{1} \frac{x}{l} \times \sin \xi_{2} \frac{x}{l} \\
& \xi_{1,2}=\sqrt{\frac{1}{2}\left[\mp \frac{B}{2 A}+\sqrt{\frac{B^{2}}{4 A^{2}}-\frac{\Delta}{4 A^{2}}}\right]}
\end{aligned}
$$


For each case the deflection $\mathrm{w}(\mathrm{x})$, the rotation of the cross section $\varphi(\mathrm{x})$, and the transverse force $\mathrm{T}(\mathrm{x})$ are determined using Equations (54), (55), and (56), respectively. The eigenvalue problem is solved and the condition for the case is checked.

\section{Results and Discussion}

\subsection{First-order vibration analysis of Timoshenko beams}

We determine herein the vibration frequencies of beams with various support conditions. The frequencies are calculated for different values of bending shear factor and coefficients of rotary inertia.

The results presented here apply for $1-\phi \alpha k_{\mathrm{RI}} \geq 0$ (low-frequency modes).

The details of the analysis and results are listed in Appendix A and in the supplementary files "Vibration analysis of a pinned-pinned beam", "Vibration analysis of a fixed-pinned beam", "Vibration analysis of a fixed-free beam", and "Vibration analysis of a fixed-fixed beam".

The vibration frequency is $\omega$, where

$$
\omega=\sqrt{\phi} \times \sqrt{\frac{E I}{\mu l^{4}}}=\lambda \times \sqrt{\frac{E I}{\mu l^{4}}} .
$$

The coefficients $\lambda$ are listed in Table 1 below.

Table 1. Natural frequencies (first mode) of Timoshenko single-span beams with various boundary conditions.

\begin{tabular}{|c|c|c|c|c|}
\hline \multicolumn{5}{|c|}{ Pinned-pinned beam } \\
\hline & \multicolumn{4}{|c|}{$\alpha=\mathrm{EI} / \kappa \mathrm{GAl}^{2}$} \\
\hline & 0.005 & 0.010 & 0.020 & 0.050 \\
\hline $\mathrm{k}_{\mathrm{RI}}=0.005$ & 9.4254 & 9.2284 & 8.8672 & 7.9873 \\
\hline $\mathrm{k}_{\mathrm{RI}}=0.010$ & 9.2284 & 9.0505 & 8.7206 & 7.8995 \\
\hline $\mathrm{k}_{\mathrm{RI}}=0.015$ & 9.0427 & 8.8814 & 8.5795 & 7.8130 \\
\hline \multicolumn{5}{|c|}{ Fixed-pinned beam } \\
\hline & \multicolumn{4}{|c|}{$\alpha=\mathrm{EI} / \kappa \mathrm{GAl}^{2}$} \\
\hline & 0.005 & 0.010 & 0.020 & 0.050 \\
\hline $\mathrm{k}_{\mathrm{RI}}=0.005$ & 14.1395 & 13.4088 & 12.2220 & 9.9416 \\
\hline $\mathrm{k}_{\mathrm{RI}}=0.010$ & 13.8341 & 13.1651 & 12.0560 & 9.8659 \\
\hline $\mathrm{k}_{\mathrm{RI}}=0.015$ & 13.5449 & 12.9307 & 11.8930 & 9.7895 \\
\hline \multicolumn{5}{|c|}{$\begin{array}{l}\text { Fixed-fixed beam } \\
\end{array}$} \\
\hline & \multicolumn{4}{|c|}{$\alpha=\mathrm{EI} / \kappa G A l^{2}$} \\
\hline & 0.005 & 0.010 & 0.020 & 0.050 \\
\hline $\mathrm{k}_{\mathrm{RI}}=0.005$ & 19.6360 & 18.0282 & 15.6938 & 11.9004 \\
\hline $\mathrm{k}_{\mathrm{RI}}=0.010$ & 19.2636 & 17.7840 & 15.5720 & 11.8705 \\
\hline $\mathrm{k}_{\mathrm{RI}}=0.015$ & 18.9052 & 17.5433 & 15.4493 & 11.8400 \\
\hline
\end{tabular}

For the case of a fixed-free beam, the results obtained in the present study are compared to those obtained by Kruszewski [6] in Table 2 below. 
Table 2. Natural frequencies (first mode) of a Timoshenko fixed-free beam.

\begin{tabular}{ccccc}
\hline \multicolumn{5}{c}{ Fixed-free beam: Present study } \\
\hline & \multicolumn{5}{c}{$\alpha=\mathrm{EI} / \kappa_{\mathrm{KAl}}{ }^{2}$} \\
& 0.005 & 0.010 & 0.020 & 0.050 \\
\cline { 2 - 5 } $\mathrm{k}_{\mathrm{RI}}=0.005$ & 3.4378 & 3.4009 & 3.3301 & 3.1394 \\
$\mathrm{k}_{\mathrm{RI}}=0.010$ & 3.4009 & 3.3659 & 3.2985 & 3.1159 \\
$\mathrm{k}_{\mathrm{RI}}=0.015$ & 3.3650 & 3.3318 & 3.2677 & 3.0927 \\
\hline \multicolumn{5}{c}{ Fixed-free beam: Kruszewski [6] } \\
\hline & 0.005 & 0.010 & 0.020 \\
\cline { 2 - 5 } & 3.4378 & 3.4009 & 3.3300 & 0.050 \\
$\mathrm{k}_{\mathrm{RI}}=0.005$ & 3.4009 & 3.3659 & 3.2985 & 3.1393 \\
$\mathrm{k}_{\mathrm{RI}}=0.010$ & 3.3650 & 3.3318 & 3.2677 & 3.1159 \\
$\mathrm{k}_{\mathrm{RI}}=0.015$ & \multicolumn{4}{c}{${ }^{2}$}
\end{tabular}

The results are identical.

\subsection{First-order vibration analysis of a Timoshenko beam with a mass, spring, or spring-mass system at its end}

The results presented here apply for $1-\phi \alpha \mathrm{k}_{\mathrm{RI}} \geq 0$ (low-frequency modes).

Timoshenko beams with the following values of $\alpha$ and $k_{\mathrm{RI}}$ were analyzed: $\quad \alpha=0.020, \quad \mathrm{k}_{\mathrm{RI}}=0.01$.

The case of a cantilevered beam carrying a tip mass at the beam's end as represented in Figure 2 was considered.

Details of the analysis and results are listed in Appendix B and in the supplementary file "Vibration analysis of a cantilevered beam carrying a tip mass".

The coefficients $\lambda$ (Equation (59)) for the natural frequencies (the first modes) are listed in Table 3 below.

Table 3. Natural frequencies (first mode) of a Timoshenko beam carrying a tip mass at the beam's end.

$\lambda$\begin{tabular}{ccccccc}
\hline & \multicolumn{7}{c}{$\mathrm{m}_{\mathrm{p}}{ }^{*}$} \\
\hline 0.25 & 0.50 & 0.75 & 1.00 & 2.00 & 3.00 & 5.00 \\
\hline 2.3662 & 1.9375 & 1.6797 & 1.5031 & 1.1211 & 0.9330 & 0.7345 \\
\hline
\end{tabular}

Let us now analyze the case of a cantilevered beam with a tip mass and spring support as shown in Figure 3. Details of the analysis and results are listed in Appendix B and in the supplementary file "Vibration analysis of a cantilevered beam with a tip mass and spring support”. The coefficients $\lambda$ are listed in Table 4 below. 


\section{TIMOSHENKO BEAM THEORY FREE VIBRATION ANALYSIS}

Table 4. Natural frequencies (first mode) of Timoshenko beams with a tip mass and spring support.

\begin{tabular}{ccccc}
\cline { 2 - 5 } & \multicolumn{4}{c}{$\mathrm{m}_{\mathrm{p}}^{*}$} \\
$\mathrm{k}_{\mathrm{p}}{ }^{*}=0.10$ & 0.50 & 1.00 & 2.00 & 5.00 \\
$\mathrm{k}_{\mathrm{p}}{ }^{*}=0.50$ & 2.1009 & 1.6303 & 1.2160 & 0.7963 \\
$\mathrm{k}_{\mathrm{p}}{ }^{*}=1.00$ & 2.2523 & 1.7481 & 1.3041 & 0.8541 \\
$\mathrm{k}_{\mathrm{p}}{ }^{*}=3.00$ & 2.7747 & 2.1556 & 1.6087 & 1.0536 \\
$\mathrm{k}_{\mathrm{p}}{ }^{*}=10.00$ & 4.0934 & 3.1917 & 2.3850 & 1.5629 \\
$\mathrm{k}_{\mathrm{p}}{ }^{*}=100.0$ & 10.1012 & 8.6664 & 6.6854 & 4.4179 \\
$\mathrm{k}_{\mathrm{p}}{ }^{*}=500.0$ & 11.8355 & 11.7926 & 11.6374 & 12.3926 \\
\hline
\end{tabular}

It is noted that with small values of spring stiffness, the values of the natural frequencies converge towards those for the case with a tip mass (Table 3), and with high values of spring stiffness, the values of the natural frequencies converge towards those for the fixed-pinned beam (12.0560, see Table 1).

Let us now analyze the case of a cantilevered beam carrying a spring-mass system at the beam's end as shown in Figure 4. Details of the analysis and results are listed in Appendix B and in the supplementary file "Vibration analysis of a cantilevered beam carrying a spring-mass system”.

The coefficients $\lambda$ are listed in Table 5 below.

Table 5. Natural frequencies (first mode) of Timoshenko beams with a spring-mass system at the beam’s end.

\begin{tabular}{rcccc}
\cline { 2 - 5 } & \multicolumn{4}{c}{$\mathrm{m}_{\mathrm{p}}{ }^{*}$} \\
$\mathrm{k}_{\mathrm{p}}{ }^{*}=0.10$ & 3.3551 & 3.3546 & 3.3544 & 3.3542 \\
$\mathrm{k}_{\mathrm{p}}{ }^{*}=0.50$ & 0.9164 & 0.6505 & 0.4636 & 0.3019 \\
$\mathrm{k}_{\mathrm{p}}{ }^{*}=1.00$ & 1.1933 & 0.8519 & 0.6061 & 0.3849 \\
$\mathrm{k}_{\mathrm{p}}{ }^{*}=3.00$ & 1.5911 & 1.1662 & 0.8391 & 0.5362 \\
$\mathrm{k}_{\mathrm{p}}{ }^{*}=4.00$ & 1.6665 & 1.2322 & 0.8908 & 0.5707 \\
$\mathrm{k}_{\mathrm{p}}{ }^{*}=10.00$ & 1.8215 & 1.3794 & 1.0115 & 0.6542 \\
$\mathrm{k}_{\mathrm{p}}{ }^{*}=100.00$ & 1.9255 & 1.4897 & 1.1087 & 0.7249 \\
\hline
\end{tabular}

It is noted that with small values of spring stiffness, the values of the natural frequencies converge towards those for the fixed-free beam (3.2985, see Table 2), and with high values of spring stiffness, the values of the natural frequencies converge towards those for the case with the tip mass (Table 3). 


\subsection{Second-order vibration analysis of Timoshenko beams}

We now determine the dynamic response of beams subjected to an axial load. An elastic Winkler foundation was not considered. A pinned-pinned beam and a fixed-pinned beam were considered.

We set the following values of $\alpha$ and $\mathrm{k}_{\mathrm{RI}}: \quad \alpha=0.020, \quad \mathrm{k}_{\mathrm{RI}}=0.01$.

The natural frequencies (first mode) were determined for various values of the axial load. Table 6 below lists the results. Details of the analysis and results are listed in Appendix $C$ and in the supplementary files "Vibration analysis of a pinned-pinned beam with an axial load" and "Vibration analysis of a fixed-pinned beam with an axial load”.

Table 6. Natural frequencies (first mode) of Timoshenko beams under axial load.

\begin{tabular}{|c|c|c|c|c|c|c|}
\hline \multicolumn{7}{|c|}{ Pinned-pinned beam } \\
\hline & & & & & & \\
\hline & -5.00 & -3.00 & -1.00 & 1.00 & 3.00 & 5.00 \\
\hline \multirow[t]{4}{*}{$\lambda$} & 5.4710 & 6.9559 & 8.1749 & 9.2340 & 10.1831 & 11.0507 \\
\hline & \multicolumn{6}{|c|}{ Fixed-pinned beam } \\
\hline & \multicolumn{6}{|c|}{$\mathrm{k}$} \\
\hline & -5.00 & -3.00 & -1.00 & 1.00 & 3.00 & -5.00 \\
\hline$\lambda$ & 9.7373 & 10.7311 & 11.6328 & 12.4635 & 13.2375 & 13.9648 \\
\hline
\end{tabular}

Ghannadiasl [4] analytically solved the case of beams with various boundary conditions resting on an elastic Winkler foundation and subjected to an axial load. The case of a beam without an elastic Winkler foundation is analyzed here. The beams have the following characteristics: Poisson's ratio $v=0.25$, Timoshenko shear coefficient $\kappa=2 / 3$, and coefficient of rotary inertia $\mathrm{k}_{\mathrm{RI}}=0.01$.

$$
\begin{aligned}
& \alpha=\frac{E I}{\kappa G A L^{2}}=\frac{1}{\kappa} \times \frac{E}{G} \times \frac{I}{A L^{2}}=\frac{1}{2 / 3} \times 2 \times(1+0.25) \times 0.01=0.0375 \\
& N_{x}=-0.6 \times \pi^{2} \times \frac{E I}{L^{2}} \quad \rightarrow \mathrm{k}=-5.922
\end{aligned}
$$

Detailed results are listed in the aforementioned supplementary files.

Table 7 lists the results of Ghannadiasl [4] and those obtained in the present study.

Table 7. Free vibration of Timoshenko beams under axial load.

\begin{tabular}{ccc}
\cline { 2 - 3 } & Pinned-pinned beam & Fixed-pinned beam \\
\cline { 2 - 3 } Ghannadiasl [4] & 3.46646 & 7.32425 \\
Present study & 3.46648 & 7.32425 \\
\hline
\end{tabular}

The results are identical. 


\section{TIMOSHENKO BEAM THEORY FREE VIBRATION ANALYSIS}

\section{Conclusions}

The material law developed in this study enables closed-form solutions of first-order and second-order vibration analysis of Timoshenko beams. Vibration analysis of a beam with a spring, a mass or a spring-mass system was conducted. Systems with an intermediate spring, mass, or spring-mass system were also analyzed. For singlespan beams, closed-form formulations of matrices expressing the boundary conditions were presented (for the case where $1-\phi \alpha \mathrm{k}_{\mathrm{RI}} \geq 0$ ); the natural frequencies were determined by solving the eigenvalue problem (the determinants of the matrices were set to zero). Closed-form expressions of first-order dynamic stiffness matrices with local coordinates were also determined (for the case where $1-\phi \alpha \mathrm{k}_{\mathrm{RI}} \geq 0$ ).

Regarding the numerical results, the following can be stated:

$\checkmark$ The natural frequencies decreased with increasing bending shear factor.

$\checkmark$ The natural frequencies decreased with increasing coefficient of rotary inertia.

$\checkmark$ The natural frequencies decreased with increasing concentrated mass.

$\checkmark$ The natural frequencies increased with increasing spring stiffness. However, in the spring-mass system, with small values of spring stiffness, the frequencies first decreased.

$\checkmark$ The natural frequencies increased with increasing tensile force.

$\checkmark$ The natural frequencies decreased with increasing compressive force.

The following aspects not treated in this study could be analyzed:

$\checkmark$ Positions of discontinuity (supports, springs, hinges), stepped beams, and multi-span beams, since the closed-form expressions of bending moments, shear forces or transversal forces, rotations of the cross sections, and deflections are known.

The following aspects were not treated in this study:

$\checkmark$ Tapered beams.

$\checkmark$ Beams subjected to damping.

$\checkmark$ The significance of the frequency for $\phi \alpha k_{\mathrm{RI}}=1$.

\section{Data Availability Statement}

The data and code that support the findings of this study are available upon reasonable request.

6. Patents

Supplementary Materials: The following files were uploaded during submission:

- Vibration analysis of a pinned-pinned beam;

- Vibration analysis of a fixed-pinned beam;

- Vibration analysis of a fixed-free beam;

- Vibration analysis of a fixed-fixed beam;

- Vibration analysis of a cantilevered beam carrying a tip mass;

- Vibration analysis of a cantilevered beam with a tip mass and spring support;

- Vibration analysis of a cantilevered beam carrying a spring-mass system;

- Vibration analysis of a pinned-pinned beam with an axial load;

- Vibration analysis of a fixed-pinned beam with an axial load.

\section{Author Contributions:}

\section{Funding:}

\section{Acknowledgments:}

Conflicts of Interest: The author declares no conflict of interest. 


\section{TIMOSHENKO BEAM THEORY FREE VIBRATION ANALYSIS}

\section{Appendix A}

Pinned-pinned beam: Detailed results are listed in the supplementary file "Vibration analysis of a pinnedpinned beam”. The boundary conditions (Equations (22) and (25)) are as follows.

$$
\begin{aligned}
& M(x=0)=0 \rightarrow A_{1}+C_{1}=0 \\
& w(x=0)=0 \rightarrow-A_{1}\left(\xi_{1}^{2}+\phi k_{R I}\right)+C_{1}\left(\xi_{2}^{2}-\phi k_{R I}\right)=0 \\
& M(x=l)=0 \rightarrow A_{1} \cosh \xi_{1}+B_{1} \sinh \xi_{1}+C_{1} \cos \xi_{2}+D_{1} \sin \xi_{2}=0 \\
& w(x=l)=0 \rightarrow-A_{1}\left(\xi_{1}^{2}+\phi k_{R I}\right) \cosh \xi_{1}-B_{1}\left(\xi_{1}^{2}+\phi k_{R I}\right) \sinh \xi_{1}+ \\
& C_{1}\left(\xi_{2}^{2}-\phi k_{R I}\right) \cos \xi_{2}+D_{1}\left(\xi_{2}^{2}-\phi k_{R I}\right) \sin \xi_{2}=0
\end{aligned}
$$

Matrix notation of the boundary conditions is as follows.

$$
\begin{aligned}
& {[M] \times \vec{C}=\overrightarrow{0}} \\
& \vec{C}=\left[\begin{array}{llll}
A_{1} & B_{1} & C_{1} & D_{1}
\end{array}\right]
\end{aligned}
$$

To determine the frequency of free vibration, the determinant of the matrix $[\boldsymbol{M}]$ was set to zero. The matrix was then as follows.

\begin{tabular}{|cccc|}
\hline 1,00 & 0,00 & 1,00 & 0,00 \\
$-\xi_{1}{ }^{2}-\phi \mathrm{k}_{\mathrm{RI}}$ & 0,00 & $\xi_{2}^{2}-\phi \mathrm{k}_{\mathrm{RI}}$ & 0,00 \\
$\cosh \xi_{1}$ & $\sinh \xi_{1}$ & $\cos \xi_{2}$ & $\sin \xi_{2}$ \\
$-\left(\xi_{1}^{2}+\phi \mathrm{k}_{\mathrm{RI}}\right) \cosh \xi_{1}$ & $-\left(\xi_{1}^{2}+\phi \mathrm{k}_{\mathrm{RI}}\right) \sinh \xi_{1}$ & $\left(\xi_{2}^{2}-\phi \mathrm{k}_{\mathrm{RI}}\right) \cos \xi_{2}$ & $\left(\xi_{2}^{2}-\phi \mathrm{k}_{\mathrm{RI}}\right) \sin \xi_{2}$ \\
\hline
\end{tabular}

Fixed-pinned beam: Detailed results are listed in the supplementary file "Vibration analysis of a fixed-pinned beam" with the following matrix.

\begin{tabular}{|cccc|}
\hline$-\xi_{1}{ }^{2}-\phi \mathrm{k}_{\mathrm{RI}}$ & 0,00 & $\xi_{2}{ }^{2}-\phi \mathrm{k}_{\mathrm{RI}}$ & 0,00 \\
0,00 & $\xi_{1}\left(\xi_{1}{ }^{2}+\phi \mathrm{k}_{\mathrm{RI}}+\alpha \phi\right)$ & 0,00 & $\xi_{2}\left(-\xi_{2}{ }^{2}+\phi \mathrm{k}_{\mathrm{RI}}+\alpha \phi\right)$ \\
$\cosh \xi_{1}$ & $\sinh \xi_{1}$ & $\cos \xi_{2}$ & $\sin \xi_{2}$ \\
$-\left(\xi_{1}{ }^{2}+\phi \mathrm{k}_{\mathrm{RI}}\right) \cosh \xi_{1}$ & $-\left(\xi_{1}{ }^{2}+\phi \mathrm{k}_{\mathrm{RI}}\right) \sinh \xi_{1}$ & $\left(\xi_{2}{ }^{2}-\phi \mathrm{k}_{\mathrm{RI}}\right) \cos \xi_{2}$ & $\left(\xi_{2}{ }^{2}-\phi \mathrm{k}_{\mathrm{RI}}\right) \sin \xi_{2}$ \\
\hline
\end{tabular}

Fixed-fixed beam: Detailed results are listed in the supplementary file "Vibration analysis of a fixed-fixed beam" with the following matrix.

\begin{tabular}{|cccc|}
\hline$-\xi_{1}{ }^{2}-\phi \mathrm{k}_{\mathrm{RI}}$ & 0,00 & $\xi_{2}{ }^{2}-\phi \mathrm{k}_{\mathrm{RI}}$ & 0,00 \\
0,00 & $\xi_{1}\left(\xi_{1}{ }^{2}+\phi \mathrm{k}_{\mathrm{RI}}+\alpha \phi\right)$ & 0,00 & $\xi_{2}\left(-\xi_{2}{ }^{2}+\phi \mathrm{k}_{\mathrm{RI}}+\alpha \phi\right)$ \\
$\xi_{1}\left(\xi_{1}{ }^{2}+\phi \mathrm{k}_{\mathrm{RI}}+\alpha \phi\right) \times$ & $\xi_{1}\left(\xi_{1}{ }^{2}+\phi \mathrm{k}_{\mathrm{RI}}+\alpha \phi\right) \times$ & $\xi_{2}\left(\xi_{2}{ }^{2}-\phi \mathrm{k}_{\mathrm{RI}}-\alpha \phi\right) \sin \xi_{2}$ & $\xi_{2}\left(-\xi_{2}{ }^{2}+\phi \mathrm{k}_{\mathrm{RI}}+\alpha \phi\right) \times$ \\
$\sinh \xi_{1}$ & $\cosh \xi_{1}$ & $\cos _{2}$ & $\cos \xi_{\mathrm{RI}}$ \\
$-\left(\xi_{1}{ }^{2}+\phi \mathrm{k}_{\mathrm{RI}}\right) \cosh \xi_{1}$ & $-\left(\xi_{1}{ }^{2}+\phi \mathrm{k}_{\mathrm{RI}}\right) \sinh \xi_{1}$ & $\left(\xi_{2}{ }^{2}-\phi \mathrm{k}_{\mathrm{RI}}\right) \cos \xi_{2}$ & $\left(\xi_{2}{ }^{2}-\phi \mathrm{k}_{\mathrm{RI}}\right) \sin \xi_{2}$ \\
\hline
\end{tabular}


Fixed-free beam: Detailed results are listed in the supplementary file "Vibration analysis of a fixed-free beam" with the following matrix.

\begin{tabular}{|cccc|}
\hline$-\xi_{1}{ }^{2}-\phi \mathrm{k}_{\mathrm{RI}}$ & 0,00 & $\xi_{2}{ }^{2}-\phi \mathrm{k}_{\mathrm{RI}}$ & 0,00 \\
0,00 & $\xi_{1}\left(\xi_{1}{ }^{2}+\phi \mathrm{k}_{\mathrm{RI}}+\alpha \phi\right)$ & 0,00 & $\xi_{2}\left(-\xi_{2}{ }^{2}+\phi \mathrm{k}_{\mathrm{RI}}+\alpha \phi\right)$ \\
$\cosh \xi_{1}$ & $\sinh \xi_{1}$ & $\cos \xi_{2}$ & $\sin \xi_{2}$ \\
$\xi_{1}\left(1+\phi \mathrm{k}_{\mathrm{RI}}{ }^{2}+\right.$ & $\xi_{1}\left(1+\phi \mathrm{k}_{\mathrm{RI}}{ }^{2}+\right.$ & $\xi_{2}\left(-1-\phi \mathrm{k}_{\mathrm{RI}}{ }^{2}+\right.$ & $\xi_{2}\left(1+\phi \mathrm{k}_{\mathrm{RI}}{ }^{2}-\right.$ \\
$\left.\mathrm{k}_{\mathrm{RI}} \xi_{1}{ }^{2}\right) \sinh \xi_{1}$ & $\left.\mathrm{k}_{\mathrm{RI}} \xi_{1}{ }^{2}\right) \cosh \xi_{1}$ & $\left.\mathrm{k}_{\mathrm{RI}} \xi_{2}{ }^{2}\right) \sin \xi_{2}$ & $\left.\mathrm{k}_{\mathrm{RI}} \xi_{2}{ }^{2}\right) \cos \xi_{2}$ \\
\hline
\end{tabular}

Kruszewski [6] presented the following equation (Equation (14) in [6]) for the determination of natural frequencies:

$2-\frac{k_{B}\left(k_{S}^{2}+k_{R I}^{2}\right)}{\sqrt{1-k_{S}^{2} k_{R I}{ }^{2} k_{B}^{2}}} \sin k_{B} \beta \sinh k_{B} \alpha+\left[k_{B}^{2}\left(k_{S}^{2}-k_{R I}^{2}\right)^{2}+2\right] \cos k_{B} \beta \cosh k_{B} \alpha=0$

where the parameters $\mathrm{k}_{\mathrm{B}}, \mathrm{k}_{\mathrm{s}}, \mathrm{k}_{\mathrm{RI}}$, $\alpha$, and $\beta$ are defined in [6] in Equations (3a), (3bi), (3c), and (5).

The following equivalences were noted between the parameters considered by Kruszewski [6] and those considered in the present study (PS):

$\mathrm{k}_{\mathrm{S}}^{2}=\alpha, \mathrm{k}_{\mathrm{B}}=\lambda, \mathrm{k}_{\mathrm{RI}}^{2}(\mathrm{~K})=\mathrm{k}_{\mathrm{RI}}(\mathrm{PS})$

\section{Appendix B}

The case of a cantilevered beam carrying a tip mass at the beam's end was considered. Detailed results are listed in the supplementary file "Vibration analysis of a cantilevered beam carrying a tip mass".

The boundary conditions (Equations (22), (25), (26), (27), and (29)) were as follows.

$$
\begin{aligned}
& w(x=0)=0 \rightarrow-\left(\xi_{1}^{2}+\phi k_{R I}\right) A_{1}+\left(\xi_{2}^{2}-\phi k_{R I}\right) C_{1}=0 \\
& \varphi(x=0)=0 \rightarrow \xi_{1}\left(\xi_{1}^{2}+\phi k_{R I}+\phi \alpha\right) B_{1}+\xi_{2}\left(-\xi_{2}^{2}+\phi k_{R I}+\phi \alpha\right) D_{1}=0 \\
& M(x=l)=0 \rightarrow A_{1} \cosh \xi_{1}+B_{1} \sinh \xi_{1}+C_{1} \cos \xi_{2}+D_{1} \sin \xi_{2}=0 \\
& \phi E I \frac{1}{l^{2}} \times w(x=l)=\phi W_{i}=-A_{1}\left(\xi_{1}^{2}+\phi k_{R I}\right) \cosh \xi_{1}-B_{1}\left(\xi_{1}^{2}+\phi k_{R I}\right) \sinh \xi_{1}+ \\
& C_{1}\left(\xi_{2}^{2}-\phi k_{R I}\right) \cos \xi_{2}+D_{1}\left(\xi_{2}^{2}-\phi k_{R I}\right) \sin \xi_{2} \\
& \left(1-\phi \alpha k_{R I}\right) \times l V_{i}=\xi_{1}\left(1+\phi k_{R I}^{2}+k_{R I} \xi_{1}^{2}\right) A_{1} \sinh _{1}+\xi_{1}\left(1+\phi k_{R I}^{2}+k_{R I} \xi_{1}^{2}\right) B_{1} \cosh \xi_{1} \\
& +\xi_{2}\left(-1-\phi k_{R I}^{2}+k_{R I} \xi_{2}^{2}\right) C_{1} \sin _{2}+\xi_{2}\left(1+\phi k_{R I}^{2}-k_{R I} \xi_{2}^{2}\right) D_{1} \cos \xi_{2} \\
& I V_{i}-m_{p}^{*} \phi W_{i}=0
\end{aligned}
$$


The boundary conditions can be expressed as in Equation (A5):

$$
\vec{C}=\left[\begin{array}{llllll}
A_{1} & B_{1} & C_{1} & D_{1} & W_{i} & l V_{i}
\end{array}\right]
$$

The determinant of the matrix $[\boldsymbol{M}]$ was set to zero. The matrix was then as follows.

$$
\left[\begin{array}{cccccc}
-\left(\xi_{1}^{2}+\phi k_{R I}\right) & 0 & \left(\xi_{2}^{2}-\phi k_{R I}\right) & 0 & 0 & 0 \\
0 & \xi_{1}\left(\xi_{1}^{2}+\phi k_{R I}+\phi \alpha\right) & 0 & \xi_{2}\left(-\xi_{2}^{2}+\phi k_{R I}+\phi \alpha\right) & 0 & 0 \\
\cosh \xi_{1} & \sinh \xi_{1} & \cos \xi_{2} & \sin \xi_{2} & 0 & 0 \\
-\left(\xi_{1}^{2}+\phi k_{R I}\right) \cosh \xi_{1} & -\left(\xi_{1}^{2}+\phi k_{R I}\right) \sinh \xi_{1} & \left(\xi_{2}^{2}-\phi k_{R I}\right) \cos \xi_{2} & \left(\xi_{2}^{2}-\phi k_{R I}\right) \sin \xi_{2} & -\phi & 0 \\
\xi_{1}\left(1+\phi k_{R I}{ }^{2}+k_{R I} \xi_{1}^{2}\right) & \xi_{1}\left(1+\phi k_{R I}{ }^{2}+k_{R I} \xi_{1}^{2}\right) & \xi_{2}\left(-1-\phi k_{R I}{ }^{2}+k_{R I} \xi_{2}^{2}\right) & \xi_{2}\left(1+\phi k_{R I}{ }^{2}-k_{R I} \xi_{2}^{2}\right) & 0 & -\left(1-\phi \alpha k_{R I}\right) \\
\times \sinh \xi_{1} & \times \cosh \xi_{1} & \times \sin \xi_{2} & \times \cos \xi_{2} & & -m_{p}^{*} \phi
\end{array}\right]
$$

The case of a tip mass and spring support as represented in Figure 3 was considered. Detailed results are listed in the supplementary file "Vibration analysis of a cantilevered beam with a tip mass and spring support".

The boundary conditions (Equations (B1), (B2), (B3), (B4), and (B5)) were applied. Equation (B6) was replaced by Equation (30).

The boundary conditions can be expressed as in Equations (A5) and (B7).

The determinant of the matrix $[\boldsymbol{M}]$ was set to zero. The matrix was then as follows.

$$
\left[\begin{array}{cccccc}
-\left(\xi_{1}^{2}+\phi k_{R I}\right) & 0 & \left(\xi_{2}^{2}-\phi k_{R I}\right) & 0 & 0 & 0 \\
0 & \xi_{1}\left(\xi_{1}^{2}+\phi k_{R I}+\phi \alpha\right) & 0 & \xi_{2}\left(-\xi_{2}^{2}+\phi k_{R I}+\phi \alpha\right) & 0 & 0 \\
\cosh \xi_{1} & \sinh \xi_{1} & \cos \xi_{2} & \sin \xi_{2} & 0 & 0 \\
-\left(\xi_{1}^{2}+\phi k_{R I}\right) \cosh \xi_{1} & -\left(\xi_{1}^{2}+\phi k_{R I}\right) \sinh \xi_{1} & \left(\xi_{2}^{2}-\phi k_{R I}\right) \cos \xi_{2} & \left(\xi_{2}^{2}-\phi k_{R I}\right) \sin \xi_{2} & -\phi & 0 \\
\xi_{1}\left(1+\phi k_{R I}{ }^{2}+k_{R I} \xi_{1}^{2}\right) & \xi_{1}\left(1+\phi k_{R I}{ }^{2}+k_{R I} \xi_{1}^{2}\right) & \xi_{2}\left(-1-\phi k_{R I}{ }^{2}+k_{R I} \xi_{2}^{2}\right) & \xi_{2}\left(1+\phi k_{R I}{ }^{2}-k_{R I} \xi_{2}^{2}\right) & 0 & -\left(1-\phi \alpha k_{R I}\right) \\
\times \sinh \xi_{1} & \times \cosh \xi_{1} & \times \sin \xi_{2} & \times \cos \xi_{2} & & 1
\end{array}\right]
$$

The case of a cantilevered beam carrying a spring-mass system at the beam's end as represented in Figure 4 was considered. Detailed results are listed in the supplementary file "Vibration analysis of a cantilevered beam carrying a spring-mass system”.

The boundary conditions (Equations (B1), (B2), (B3), (B4), and (B5)) were applied. Equation (B6) was replaced by Equations (31a) and (31b).

The boundary conditions can be expressed as in Equation (A5):

$$
\vec{C}=\left[\begin{array}{lllllll}
A_{1} & B_{1} & C_{1} & D_{1} & W_{i} & l V_{i} & W_{i M}
\end{array}\right]
$$


The determinant of the matrix $[\boldsymbol{M}]$ was set to zero. The matrix was then as follows.

$\left[\begin{array}{ccccccc}-\left(\xi_{1}^{2}+\phi k_{R I}\right) & 0 & \left(\xi_{2}^{2}-\phi k_{R I}\right) & 0 & 0 & 0 & 0 \\ 0 & \xi_{1}\left(\xi_{1}^{2}+\phi k_{R I}+\phi \alpha\right) & 0 & \xi_{2}\left(-\xi_{2}^{2}+\phi k_{R I}+\phi \alpha\right) & 0 & 0 & 0 \\ \cosh \xi_{1} & \sinh \xi_{1} & \cos \xi_{2} & \sin \xi_{2} & 0 & 0 & 0 \\ -\left(\xi_{1}^{2}+\phi k_{R I}\right) \cosh \xi_{1} & -\left(\xi_{1}^{2}+\phi k_{R I}\right) \sinh \xi_{1} & \left(\xi_{2}^{2}-\phi k_{R I}\right) \cos \xi_{2} & \left(\xi_{2}^{2}-\phi k_{R I}\right) \sin \xi_{2} & -\phi & 0 & 0 \\ \xi_{1}\left(1+\phi k_{R I}^{2}+k_{R I} \xi_{1}^{2}\right) & \xi_{1}\left(1+\phi k_{R I}{ }^{2}+k_{R I} \xi_{1}^{2}\right) & \xi_{2}\left(-1-\phi k_{R I}^{2}+k_{R I} \xi_{2}^{2}\right) & \xi_{2}\left(1+\phi k_{R I}^{2}-k_{R I} \xi_{2}^{2}\right) & 0 & -\left(1-\phi \alpha k_{R I}\right) & 0 \\ \times \sinh \xi_{1} & \times \cosh \xi_{1} & \times \sin \xi_{2} & \times \cos \xi_{2} & & \\ 0 & 0 & 0 & 0 & 0 & 1 & -m_{p}^{*} \phi \\ 0 & 0 & 0 & 0 & -k_{p}^{*} & 0 & \left(k_{p}^{*}-m_{p}^{*} \phi\right)\end{array}\right]$

\section{Appendix C}

Without a Winkler foundation, the solution to Equation (61) is as follows (Equations (66c) and (66d)).

$$
\begin{aligned}
& M(x)=A_{1} \cosh \xi_{1} \frac{x}{l}+B_{1} \sinh \xi_{1} \frac{x}{l}+C_{1} \cos \xi_{2} \frac{x}{l}+D_{1} \sin \xi_{2} \frac{x}{l} \\
& \xi_{1,2}=\sqrt{(\mp B+\sqrt{\Delta}) / 2 A}
\end{aligned}
$$

The parameters A, B, and $\Delta$ are defined in Equations (65a), (65b), and (65d), respectively.

Substituting Equations (C1) and (65a) to (65e) into Equations (62) and (63) yields the following.

$$
\begin{aligned}
& \phi E I / l^{2} \times w(x)=\left(-A \xi_{1}^{2}-E\right) A_{1} \cosh \xi_{1} \frac{x}{l}+\left(-A \xi_{1}^{2}-E\right) B_{1} \sinh \xi_{1} \frac{x}{l} \\
& +\left(A \xi_{2}^{2}-E\right) C_{1} \cos \xi_{2} \frac{x}{l}+\left(A \xi_{2}^{2}-E\right) D_{1} \sin \xi_{2} \frac{x}{l} \\
& \phi E I / l^{2} \times\left(\phi \alpha k_{R I}-1\right) \times l \varphi(x)=\left(B \xi_{1}+A \xi_{1}^{3}\right) A_{1} \sinh \xi_{1} \frac{x}{l} \\
& +\left(B \xi_{1}+A \xi_{1}^{3}\right) B_{1} \cosh \xi_{1} \frac{x}{l}+\left(-B \xi_{2}+A \xi_{2}^{3}\right) C_{1} \sin \xi_{2} \frac{x}{l}+\left(B \xi_{2}-A \xi_{2}^{3}\right) D_{1} \cos \xi_{2} \frac{x}{l}
\end{aligned}
$$




\section{TIMOSHENKO BEAM THEORY FREE VIBRATION ANALYSIS}

For the pinned-pinned beam, the determinant of the matrix expressing the boundary conditions $(M(x=0)=0$; $\mathrm{w}(\mathrm{x}=0)=0 ; \mathrm{M}(\mathrm{x}=\mathrm{l})=0 ; \mathrm{w}(\mathrm{x}=\mathrm{l})=0)$ was set to zero. The matrix was then as follows.

\begin{tabular}{|cccc|}
\hline 1,00 & 0,00 & 1,00 & 0,00 \\
$-\mathrm{A} \xi_{1}^{2}-\mathrm{E}$ & 0,00 & $\mathrm{~A} \xi_{2}^{2}-\mathrm{E}$ & 0,00 \\
$\cosh \xi_{1}$ & $\sinh \xi_{1}$ & $\cos \xi_{2}$ & $\sin \xi_{2}$ \\
$\left(-\mathrm{A} \xi_{1}{ }^{2}-\mathrm{E}\right) \cosh \xi_{1}$ & $\left(-\mathrm{A} \xi_{1}{ }^{2}-\mathrm{E}\right) \sinh \xi_{1}$ & $\left(\mathrm{~A} \xi_{2}{ }^{2}-\mathrm{E}\right) \cos \xi_{2}$ & $\left(\mathrm{~A} \xi_{2}{ }^{2}-\mathrm{E}\right) \sin \xi_{2}$ \\
\hline
\end{tabular}

For the fixed-pinned beam, the determinant of the matrix expressing the boundary conditions $(\mathrm{w}(\mathrm{x}=0)=0$; $\varphi(\mathrm{x}=0)=0 ; \mathrm{M}(\mathrm{x}=\mathrm{l})=0 ; \mathrm{w}(\mathrm{x}=\mathrm{l})=0$ ) was set to zero. The matrix was then as follows.

\begin{tabular}{|cccc|}
\hline$-\mathrm{A} \xi_{1}{ }^{2}-\mathrm{E}$ & 0,00 & $\mathrm{~A} \xi_{2}{ }^{2}-\mathrm{E}$ & 0,00 \\
0,00 & $\mathrm{~B} \xi_{1}+\mathrm{A} \xi_{1}{ }^{3}$ & 0,00 & $\mathrm{~B} \xi_{2}-\mathrm{A} \xi_{2}{ }^{3}$ \\
$\cosh \xi_{1}$ & $\sinh \xi_{1}$ & $\cos \xi_{2}$ & $\sin \xi_{2}$ \\
$\left(-\mathrm{A} \xi_{1}{ }^{2}-\mathrm{E}\right) \cosh \xi_{1}$ & $\left(-\mathrm{A} \xi_{1}{ }^{2}-\mathrm{E}\right) \sinh \xi_{1}$ & $\left(\mathrm{~A} \xi_{2}{ }^{2}-\mathrm{E}\right) \cos \xi_{2}$ & $\left(\mathrm{~A} \xi_{2}{ }^{2}-\mathrm{E}\right) \sin \xi_{2}$ \\
\hline
\end{tabular}

\section{References}

[1] T. Kocatürk, M. Simsek: Free vibration analysis of Timoshenko beams under various boundary conditions. Journal of Engineering and Natural Sciences, January 2005

[2] M. Gürgöze: On the eigenfrequencies of a cantilever beam with attached tip mass and a spring-mass system. Journal of Sound and Vibration (1996) 190(2), 149-162. https://doi.org/10.1006/jsvi.1996.0053

[3] Y. Yesilce, O. Demirdag, S. Catal: Free vibrations of a multi-span Timoshenko beam carrying multiple spring-mass systems. Sadhana Vol. 33, Part 4, August 2008, pp. 385-401.

https://doi.org/10.1007/s12046-008-0026-1.

[4] A. Ghannadiasl, M. Mofid: An analytical solution for free vibration of elastically restrained Timoshenko beam on an arbitrary variable Winkler foundation and under axial load. Lat. Am. J. Solids Struct. 12 (2015) 2417-2438. https://doi.org/10.1590/1679-78251504

[5] N. N. Osadebe, J.C. Agunwamba, M.E. Onyia, E.O. Rowland-Lato: Free Vibration Analysis of Timoshenko Beam Using Energy Separation Principle. IJSER vol 9, Issue 2, February 2018

[6] Kruszewski, Edwin T: Effect of transverse shear and rotary inertia on the natural frequency of a uniform beam. National advisory committee for aeronautics, Technical note 1909 https://ntrs.nasa.gov/search.jsp?R=19930082587 